\title{
Use of COSMO-RS for the prediction of adsorption equilibria
}

\author{
C. Mehler, A. Klamt, W. Peukert
}

\begin{abstract}
The novel method COSMO-RS for the calculation of thermodynamic partition equilibria based on quantum chemical calculations is for the first time applied to the correlation and prediction of adsorption equilibria. In the limit of small adsorbate concentrations very good predictions have been achieved for adsorption equilibria of activated carbon from gas phase as well as from aqueous phase.
\end{abstract}

\section{Introduction}

In the last years the adsorption from the liquid phase has gained increasingly in importance. The knowledge of the adsorption equilibria is necessary for the sizing of adsorption plants, as for example for the waste water treatment (Sontheimer (1985)) or for the adsorptive removal of trace components from liquids (e.g. water from THF). Preferentially used adsorbents are activated carbon and carbon blacks, because they are cheap and have a relatively high sorption capacity. But the adsorption is also an important step in many applications like the crystallization, the heterogeneous catalysis, the stabilization of nano-particles and of re-dispersible agglomerates. Therefore the elucidation of this adsorption step enables a better explanation of the processes specified above. As experimental studies of the adsorption behavior are very expensive and time-consuming it is tried to predict adsorption equilibria only by the physical properties of adsorbate, adsorbent and solvent. At present interactions between fluid molecules can be calculated but due to the complex structure of solid surfaces this is not possible to characterize them.

With the increasing performance of computers, a new strategy for the prediction of thermophysical data came up in the last years: For molecules in the gas-phase quantum chemical methods, and especially density functional methods (DFT), have become a reliable and efficient tool for the calculation of the molecular properties. If combined with dielectric continuum solvation methods (for reviews see: Cramer (1995) and Tomasi (1994)) these methods can be used for the calculation of free energies and other properties of molecules in solvents, as well. The Conductor-Like Screening Model (COSMO) presented by Klamt et. al. in 1993 is a highly efficient variant of these continuum solvation methods. In 1995 Klamt generalized the concept 
of COSMO to a more realistic treatment of solvents (COSMO-RS) by a combination of molecular surface interaction energies derived from COSMO with an efficient and accurate statistical thermodynamics (Klamt (1995)). Thus COSMO-RS for the first time allows the predictive calculation of vapor pressures, activity coefficients, and phase equilibria based on quantum chemical calculations. The only information that is needed for the calculation is the molecular structure of the concerned components. Basically, these calculations are suited for pure and mixed fluids of known composition, but they have been successfully applied to polymers, as well. Thermophysical data of systems including fluids of chemically less well defined composition like blood, or including heterogeneous solids of unknown surface composition, like the industrially used adsorbates, cannot be directly calculated. Nevertheless, there is an indirect way to treat such systems by COSMO-RS. The scope of this paper is to demonstrate, how COSMO-RS can be used for the prediction of solid-liquid and solid-gas equilibria involving adsorbates.

\section{Conductor-Like Screening Model for Real Solvents}

COSMO-RS is a novel theory combining the concepts of quantum theory, dielectric continuum models, surface interactions, and of statistical thermodynamics. Since a full derivation of the theory of COSMO-RS is beyond the scope of this article, a short summary of the essentials will be given here. The reader interested in details is referred to the references.

COSMO-RS considers a liquid system as an ensemble of molecules of different kinds, including solvent and solute. For each kind of molecules $X$ a density functional (DFT) COSMO calculation is performed in order to get the total energy $E^{X}$ cosmo and the polarization (or screening) charge density (SCD) $\sigma$ on its molecular surface. $\sigma$ is an extremely good and local descriptor of molecular surface polarity.

For the purpose of an efficient statistical thermodynamics calculation the liquid ensemble of molecules now is considered as an ensemble of pair-wise interacting molecular surfaces. The most important parts of the specific interaction between molecular surfaces, i.e. electrostatics (es) and hydrogen bonding (hb), are expressed by the SCDs $\sigma$ and $\sigma^{\prime}$ of the contacting surface pieces:

$$
\mathrm{E}_{\mathrm{es}}\left(\sigma, \sigma^{\prime}\right)=\frac{\alpha^{\prime}}{2}\left(\sigma+\sigma^{\prime}\right)^{2}
$$

and

$$
\mathrm{E}_{\mathrm{hb}}\left(\sigma, \sigma^{\prime}\right)=\mathrm{c}_{\mathrm{hb}} \min \left\{0, \sigma \sigma^{\prime}+\sigma_{\mathrm{hb}}{ }^{2}\right\}
$$


The three parameters $\alpha^{\prime}, \mathrm{Chb}$, and ohb have been adjusted to a large number of thermodynamic data. Since all relevant interactions depend on $\sigma$, the distribution functions (histograms) $p^{x}(\sigma)$ are required for the statistical thermodynamics. These " $\sigma$-profiles" can easily be derived from the COSMO output. Note, that the $\sigma$-profiles provide a vivid picture of the molecular polarity (see Figure 1, and a discussion given by Klamt (1995) and Klamt (1998)). Furthermore we need the $\sigma$-profile $\operatorname{ps}(\sigma)$ of the ensemble S. For multicomponent systems $\operatorname{ps}(\sigma)$ is simply calculated as a sum of the molecular $\sigma$-profiles of the concerned components $X_{i}$ weighted by the mol-fractions $\mathrm{x}_{\mathrm{i}}$ :

$p_{s}(\sigma)=\frac{\sum_{i} x_{i} p^{x_{i}}(\sigma)}{\sum_{i} x_{i}}$

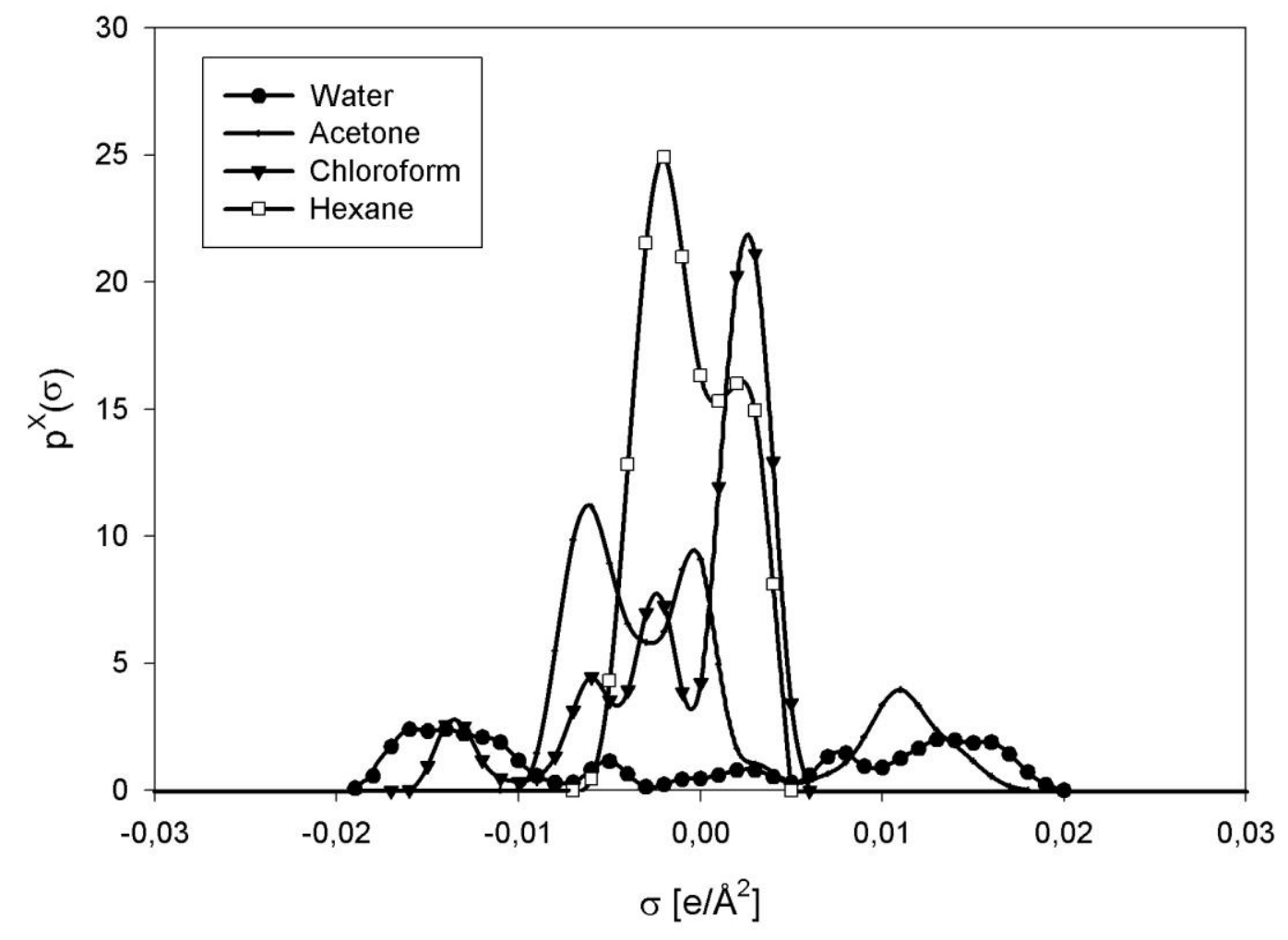

Figure 1: $\sigma$-profiles of different solvents 
Now the chemical potentials of the compounds in the solvent are calculated by a novel, exact and very efficient statistical thermodynamics procedure. The first step is the iterative solution of the equation

$$
\mu_{\mathrm{S}}(\sigma)=-\frac{R T}{a_{\text {eff }}} \ln \left\{\int p_{S}\left(\sigma^{\prime}\right) \exp \left(\frac{a_{\text {eff }}}{R T}\left(\mu_{S}\left(\sigma^{\prime}\right)-E\left(\sigma, \sigma^{\prime}\right)\right)\right) d \sigma^{\prime}\right\}
$$

This implicit equation, in which aeff denotes an effectively independent piece of molecular area, can be solved by iteration within milliseconds on a PC. It yields the function $\mu \mathrm{s}(\sigma)$ ( $\sigma$-potential) which tells how much the solvent $S$ likes surface of polarity $\sigma$. This is a very characteristic function for each solvent. We call it the $\sigma$ potential of solvent $\mathrm{S}$. Examples are given in Figure 2.

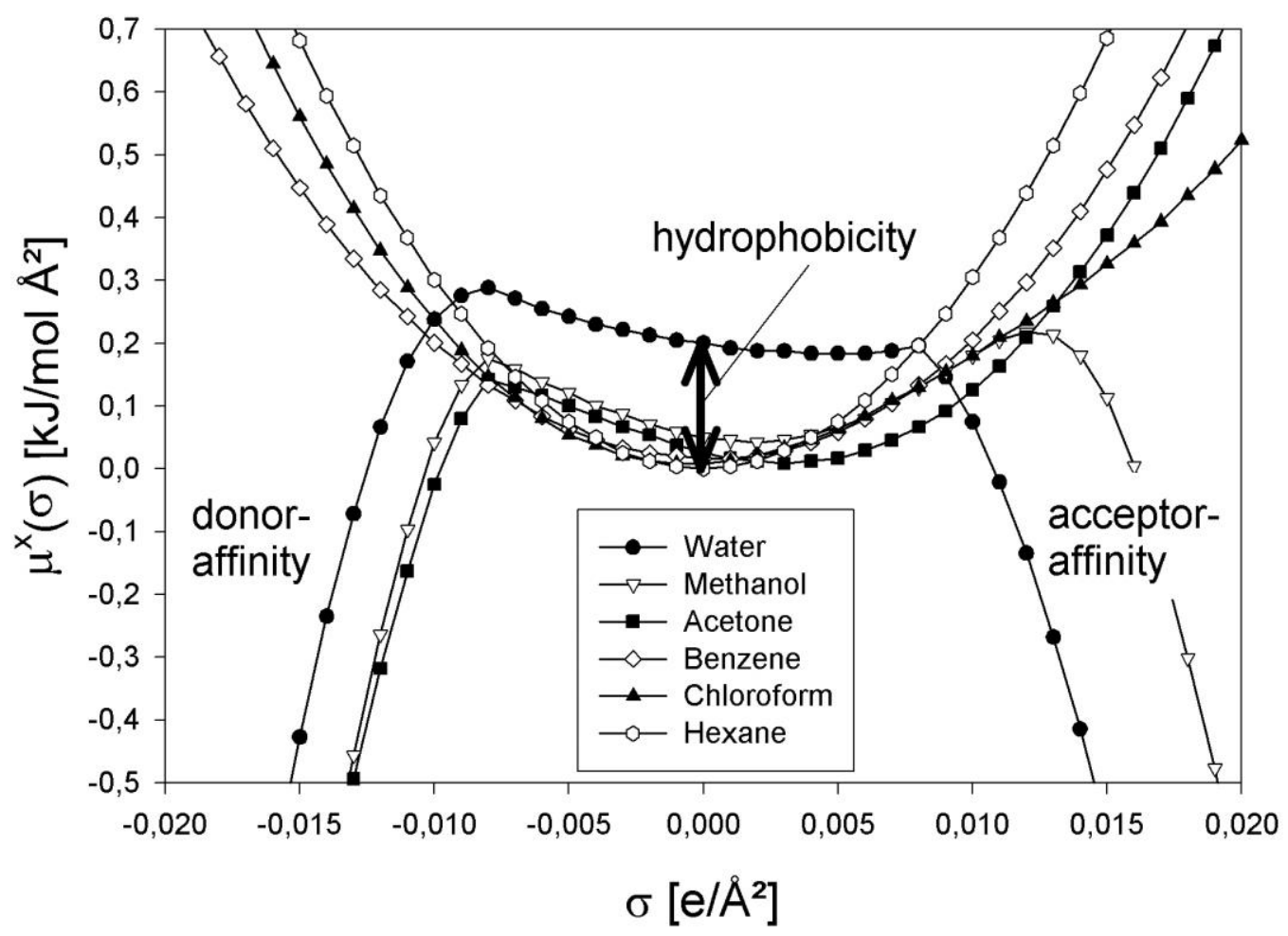

Figure 2: $\sigma$-potentials of solvents

These $\sigma$-potentials describe the solvent behavior regarding electrostatics, HB-affinity, and hydrophobicity. In a second step the $\sigma$-potential is integrated over the surface of each compound $X$, yielding the chemical potential of $X$ in $S$ : 
$\mu_{S}^{X}=\int p^{X}(\sigma) \mu_{S}(\sigma) d \sigma+\mu_{\text {combs }}^{X}$

In this equation the surface integral is evaluated as an $\sigma$-integral, making use of the $\sigma$-profile of the solute $X$. The combinatorial contribution $\mu^{X}$ comb,s to $\mu$ takes into account size and shape effects of solute and solvent (Klamt (2000)). Usually it is small compared to the first term in eq. 4 which results from the surface interactions. It is sufficient to consider it as a solvent specific constant, here.

As a result of this series of relatively simple steps, starting from a quantum chemical calculation for each compound we found an expression for the chemical potential of an almost arbitrary chemical compound $\mathrm{X}$ in an almost arbitrary solvent $\mathrm{S}$, which may be a pure compound or a mixture. This allows us to calculate any partition coefficient as well as solubility. Based on density functional COSMO calculations, the few parameters required in COSMO-RS, have been fitted to a large set of experimental data (Klamt (1998)), covering 215 diverse chemical compounds and the properties $\Delta G_{\text {hydr, }} \log P_{\text {vapor, }}$ and the aqueous partition coefficients with octanol, hexane, benzene and ether. Note, that the properties $\Delta \mathrm{G}$ hydr and $\log \mathrm{P}_{\text {vapor }}$ involve the gasphase, which requires a small addendum to the steps given above that is not of interest here. However, since the logarithmic aqueous solubility is the difference of

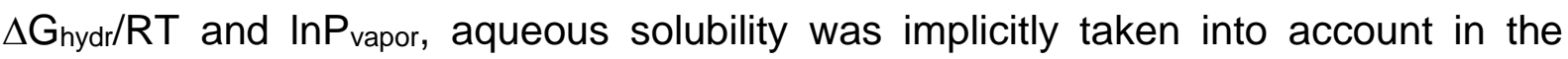
parameterization of COSMO-RS. The initial COSMO-RS parameterization yielded a rms-error of 0.3 log-units for the diverse partition and solubility properties of small and medium sized molecules. In recent parameterizations the error has been reduced to about 0.23 log-units.

\section{Extension of COSMO-RS to complex solutions and adsorbates}

As shown in Chapter 2, COSMO-RS is a reliable method for the a priory prediction of thermophysical data and phase equilibria of pure fluids and liquid mixtures of well defined composition. Nevertheless, there are several thermodynamic equilibria of industrial importance, which involve one or more phases, which are either chemically less defined, or which are disordered, but not really liquid, or both. Examples of such systems are physiological phases like blood, brain, or special tissue, structurally sophisticated polymers, and solid adsorbents, like activated carbon. Since in such phases no surface composition function $\operatorname{ps}(\sigma)$ is available, the $\sigma$-potential $\mu s(\sigma)$ of the phase $S$ and the chemical potentials $\mu \mathrm{s}^{X}$ of solutes $X$ in these phases cannot be directly calculated by COSMO-RS. But an indirect treatment of such phases by COSMO-RS is enabled by the following extension: 
Consideration of a large number of different solvents led to the finding (see as well figure 2) that $\sigma$-potentials can be described very well by a Taylor-like of the form

$$
\mu_{S}(\sigma) \cong \sum_{i=-2}^{m} c_{S}^{i} f_{i}(\sigma)
$$

with

$$
f_{i}(\sigma)=\sigma^{i} \quad \text { for } i \geq 0
$$

and

$$
f_{-2 /-1}(\sigma)=f_{\text {acc } / \text { don }}(\sigma) \cong\left\{\begin{array}{lll}
0 & \text { if } & \pm \sigma<\sigma_{h b} \\
\mp \sigma+\sigma_{h b} & \text { if } & \pm \sigma>\sigma_{h b}
\end{array}\right.
$$

The highest order of the polynomial contributions (eq. 7) required for a sufficient description of $\sigma$-potentials typically is $m=3$. The hydrogen bonding contributions expressed by eq. 8 are necessary to describe the acceptor and donor behavior of solvent. As can be seen in figure 2, this behavior corresponds to a linear descent in the $\sigma$-potentials starting from some threshold $\sigma$ hb. The functions $f_{\text {acc }}(\sigma)$ and $f_{\text {don }}(\sigma)$ are well capable of describing just these features of the $\sigma$-potentials. Using this Taylor expansion, we may characterize each solvent (at fixed temperature, usually room temperature) by the set of $\sigma$-coefficients $c^{i}$ s. Obviously any difference between the $\sigma$ potentials of two solvents is of the same kind of expansion, with coefficients $c^{i} s, S^{\prime}$ being just the difference of the coefficients of the two solvents. Partition coefficients are connected with the pseudo-chemical potentials by the equation

$$
\mathrm{kT} \ln K_{\mathrm{s}, \mathrm{s}^{\prime}}^{\mathrm{X}}=\left[\mu_{\mathrm{s}^{\prime}}^{\mathrm{x}}-\mu_{\mathrm{S}}^{\mathrm{x}}\right]
$$

If the $\sigma$-potential of the solvents and the $\sigma$-profile of the solute are known the pseudo chemical potentials $\mu_{S}^{X}$ and $\mu_{S^{\prime}}^{X}$ of the component $X$ in the solutes $S$ and $S^{\prime}$ can be calculated with eq. 5 . Using eq. 6 for $\mu_{s}(\sigma)$, we thus find that any partition coefficient between two solvents $S$ and $S^{\prime}$ should be expressible in the form

$$
\begin{aligned}
& \ln \mathrm{K}_{\mathrm{S}, \mathrm{S}^{\prime}}^{\mathrm{X}}=\frac{1}{\mathrm{kT}}\left[\mathrm{C}_{\mathrm{S}, \mathrm{S}^{\prime}}+\int \mathrm{p}^{\mathrm{X}}(\sigma)\left(\mu_{\mathrm{S}^{\prime}}(\sigma)-\mu_{\mathrm{S}}(\sigma)\right) \mathrm{d} \sigma\right] \\
& \cong \tilde{\mathrm{c}}_{S, S^{\prime}}+\int \mathrm{p}^{\mathrm{X}}(\sigma) \sum_{\mathrm{i}=-2}^{\mathrm{m}} \tilde{\mathrm{c}}_{\mathrm{S}, \mathrm{S}^{\prime}}^{\mathrm{f}} \mathrm{f}_{\mathrm{i}}(\sigma) \mathrm{d} \sigma=\tilde{\mathrm{c}}_{\mathrm{S}, \mathrm{S}^{\prime}}+\sum_{\mathrm{i}=-2}^{\mathrm{m}} \tilde{\mathrm{c}}_{\mathrm{S}, \mathrm{S}^{\prime}}^{\mathrm{i}} \mathrm{M}_{\mathrm{i}}^{\mathrm{X}}
\end{aligned}
$$


where the combinatorial contributions have been subsumed in $\tilde{\mathrm{c}}_{\mathrm{S}, \mathrm{S}^{\prime}}$ and the $\sigma$ moments $\mathrm{Mi}^{\mathrm{X}}$ of the solute $\mathrm{X}$ are defined by

$$
M_{i}^{X}=\int p^{X}(\sigma) f_{i}(\sigma) d \sigma
$$

Thus with eqs. 6 and 10 the $\sigma$-potentials of any solvent (or solid) can be approximately calculated by using the linear regression of the partition coefficients of different solutes with their $\sigma$-moments. Eq. 11 implies that any logarithmic partition coefficient can be represented as a linear combination of $\sigma$-moments. As a consequence, the set of $\sigma$-moments $\mathrm{Mi}^{\mathrm{X}}, \mathrm{i}=0,2,3$, complemented by the hydrogen bond moments $\mathrm{Macc}^{\mathrm{X}}\left(=\mathrm{M}-2^{\mathrm{X}}\right)$ and $\mathrm{Mdon}^{\mathrm{X}}\left(=\mathrm{M}_{-1} \mathrm{X}\right)$ should be a very good and almost complete set of molecular descriptors for a linear regression analysis of any partition problem. Note, that the first moment $\mathrm{M}_{1} \mathrm{x}$ usually is of no importance, because it is just the negative of the total charge of the molecule. Hence, for neutral compounds $\mathrm{M}_{1} \mathrm{X}$ trivially vanishes. By definition of the $\sigma$-profiles the zero-th moment $\mathrm{M}_{0} \mathrm{X}$ is identical with the molecular surface. The second moment is an excellent measure of the overall polarity of the solute, and the third moment is a measure of the asymmetry of the sigma profile. The hydrogen bond moments are quantitative measure of the acceptor and donor capacities of the compound $\mathrm{X}$, respectively.

\section{Application to adsorption equilibria}

In the first step, we just regard adsorption from dilute aqueous solutions, because in the very low concentration range, one can assume, that the interactions between the adsorbed molecules are due to their spatial distance very small and the change of composite sigma profiles can be neglected. On heterogeneous adsorbents like activated carbon with energetically different adsorption sites, first the energetically higher sites will be occupied and with increasing concentration, the energetically lower sites will be used step by step. Thus a curved shape of the isotherm is expected. Nevertheless - in the scope of the precision of - in the very low concentration range a linear increase of the adsorbed amount $\mathrm{n}^{\mathrm{x}}$ with increasing concentration $\mathrm{c}^{\mathrm{X}}$ can be detected (see Fig. 3).

$$
\mathrm{n}^{\mathrm{x}}=\mathrm{H}^{\mathrm{x}} \cdot \mathrm{c}^{\mathrm{x}}
$$




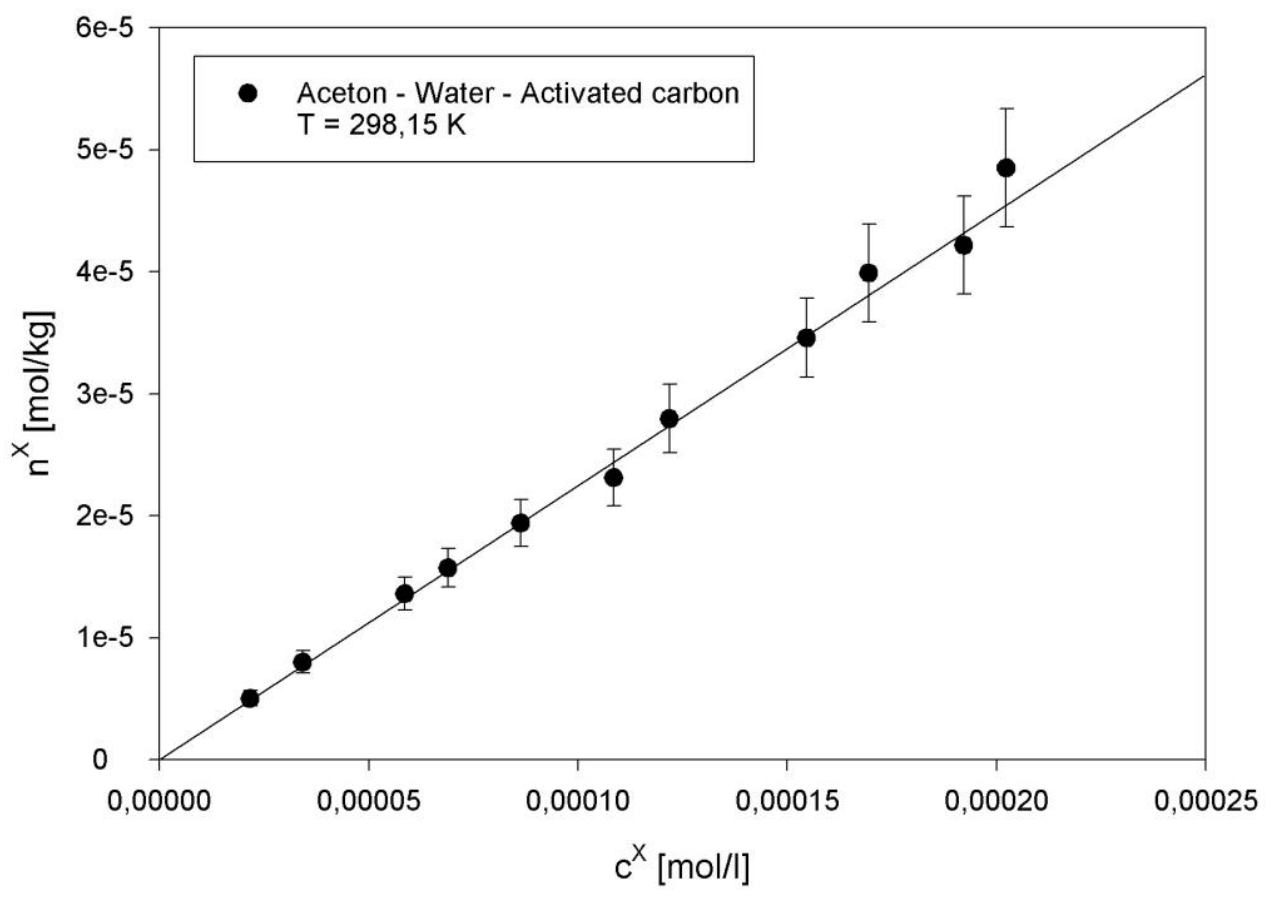

Figure 3: Adsorption isotherme of acetone from aqueous solution on activated carbon, $T=298,15 \mathrm{~K}$; assumed standard error: $10 \%$

Thus the adsorption can be described with one parameter $\mathrm{H}^{\mathrm{x}}$. Analogous to the adsorption from gaseous phase, this parameter is sometimes called averaged Henry's Law coefficient.

With

$$
\begin{aligned}
& \mu_{S 1}^{x_{i}}=\mu_{0}^{x_{i}}+k T \ln \gamma_{S 1}^{x_{1}} \\
& \mu_{S 2}^{x_{i}}=\mu_{0}^{X_{i}}+k T \ln \gamma_{S 2}^{X}
\end{aligned}
$$

and

$$
\frac{x_{S 1}^{x}}{x_{S 2}^{x}}=\frac{\gamma_{S 2}^{x}}{\gamma_{S 1}^{x}}
$$

the equation for the calculation of $H^{x}$ follows:

$$
H^{x}=\frac{n^{X}}{c^{X}}=\frac{\tilde{M}_{S 1} \rho_{S 1}}{\tilde{M}_{S 2}} \frac{x_{S 2}^{X}}{x_{S 1}^{x}}=\frac{\tilde{M}_{S 1} \rho_{S 1}}{\tilde{M}_{S 2}} \frac{\gamma_{S 1}^{X}}{\gamma_{S 2}^{x}}=\frac{\tilde{M}_{S 1} \rho_{S 1}}{\tilde{M}_{S 2}} \exp \left(\frac{\mu_{S 1}^{x_{i}}-\mu_{S 2}^{x_{i}}}{k T}\right)
$$


$\gamma_{\mathrm{S} 1}^{\mathrm{x}}$ and $\gamma_{\mathrm{S} 2}^{\mathrm{X}}$ are the activity coefficients, $\tilde{\mathrm{M}}_{\mathrm{S} 1}$ and $\tilde{\mathrm{M}}_{\mathrm{S} 2}$ are the molecular masses of the solvent and the adsorbent and $\rho_{S 1}$ is the density of the solvent. Thus besides the last mentioned molecular parameters only the charge frequency distributions from solvent, adsorbent and adsorptive $p^{s 1}(\sigma), p^{s 2}(\sigma)$ and $p^{x}(\sigma)$ are necessary for the prediction of $\mathrm{H}^{\mathrm{X}}$. Even if with the increasing performance of modern computers, it is still not possible to calculate the charge distribution of energetically heterogeneous solid surfaces $p^{\text {s2 }}$ a priori. This is mainly caused by the unknown composition and structure of heterogeneous solids. Therefore one have to characterize the solid with the help of experimental data.

\section{Correlation with the sigma-moments}

The infinite dilution adsorption coefficients $\mathrm{H}^{X}$ can be considered as partition coefficients of the adsorbates between the "absorbents phase" and a reference phase. Hence we expect that they can be described by a $\sigma$-moment regression, as described in the previous section. In this section we will discuss the use of this COSMO-RS $\sigma$-moment approach to 2 sets of adsorption coefficients, one being for the adsorption from gas-phase to activated carbon, the other for adsorption from water to activated carbon. Most of the experimental data sets, covering 19 and 24 compounds, respectively, have been collected from literature (Baldauf (1986), Chatzopoulos (1993), Urano (1982), Juang (1996), Kuramae (1986), Miyahara (1992), Radke and Prausnitz (1972), Sakoda (1986), Fukuchi (1989)), some of them are data from our own measurements. Experimental and theoretical data are given in Tables 1 and 2. Due to the lack of experimental data, we have included both results from our own measurements al well as results from literature on different active carbon materials. We are aware that activated carbon is heterogeneous due to impurities and different conditioning prior to measurements. Nevertheless, we can show that Henry coefficients both in the gas and in the liquid phase can be predicted surprisingly well by our model. Currently measurements are carried out with specific activated carbon and other materials which will allow us to distinguish between individual solids and will further improve our predictions. This paper introduces the new method and proves it's applicability on the basis of the existing experimental data.

Tab. 1: Adsorption from gaseous phase: Experimental data ( $T=300 \mathrm{~K}$, Adsorbent: Activated Carbon) and sigma moments of components $X$
Adsorptive
$\log \frac{H_{i}(\text { th. })}{m^{3} / k g P a}$
$\mathrm{M}^{\mathrm{x}}{ }_{0}$
$\mathrm{M}_{2}$
$\mathbf{M}^{\mathrm{X}}{ }_{\text {acc }}$
$\mathbf{M}_{\text {don }}$ 


\begin{tabular}{|c|c|c|c|c|c|c|}
\hline & $\begin{array}{c}\log \frac{H_{i}(\exp .)}{\mathbf{m}^{3} / \mathbf{k g P a}} \\
{[-]}\end{array}$ & {$[-]$} & {$\left[\AA^{2}\right]$} & {$\left[10^{-38} \mathrm{C}^{2} / \AA^{4}\right]$} & {$\left[10^{-19} \mathrm{C}\right]$} & {$\left[10^{-19} \mathrm{C}\right]$} \\
\hline Acetone & $-0,69$ & $-1,23$ & 102,6792 & 122,98 & 4,48 & 0,00 \\
\hline Benzene & 0,25 & $-0,17$ & 123,6773 & 73,30 & 0,00 & 0,00 \\
\hline Carbondioxide & $-4,17$ & $-3,98$ & 66,1657 & 55,89 & 0,00 & 0,00 \\
\hline Carbonmonoxide & $-5,25$ & $-4,88$ & 57,8705 & 17,24 & 0,00 & 0,00 \\
\hline Dichloromethane & $-1,74$ & $-1,66$ & 98,4893 & 84,33 & 0,00 & 0,00 \\
\hline Diethylether & $-0,40$ & 0,13 & 130,349 & 80,69 & 4,22 & 0,00 \\
\hline Ethane & $-3,65$ & $-3,70$ & 77,1878 & 12,01 & 0,00 & 0,00 \\
\hline Ethene & $-3,56$ & $-3,74$ & 72,2284 & 40,79 & 0,00 & 0,00 \\
\hline Ethylene & $-3,82$ & $-3,47$ & 68,6006 & 93,66 & 0,00 & 0,00 \\
\hline $\mathrm{H} 2 \mathrm{~S}$ & $-3,69$ & $-3,88$ & 62,4876 & 85,14 & 0,17 & 0,15 \\
\hline Hydrogen & $-6,43$ & $-6,94$ & 27,3222 & 5,27 & 0,00 & 0,00 \\
\hline Methane & $-4,82$ & $-5,07$ & 55,6966 & 12,22 & 0,00 & 0,00 \\
\hline Methanol & $-2,17$ & $-2,05$ & 66,3616 & 137,55 & 6,77 & 3,43 \\
\hline Nitrogen & $-5,43$ & $-5,11$ & 54,7439 & 14,22 & 0,00 & 0,00 \\
\hline Propane & $-2,56$ & $-2,41$ & 97,3749 & 13,99 & 0,00 & 0,00 \\
\hline Propene & $-2,43$ & $-2,57$ & 90,3286 & 43,66 & 0,00 & 0,00 \\
\hline Trichloroethene & 0,34 & 0,05 & 130,9097 & 47,74 & 0,00 & 0,00 \\
\hline Trifluromethane & $-3,52$ & $-2,97$ & 80,2262 & 68,68 & 0,00 & 0,00 \\
\hline Water & $-1,87$ & $-1,93$ & 43,0598 & 193,29 & 9,23 & 6,18 \\
\hline
\end{tabular}

Standard deviation: 0,377

Tab. 2: Adsorption from aqueous phase: Experimental data ( $T=300 \mathrm{~K}$, Adsorbent: Activated Carbon) and sigma moments of components $X$

\begin{tabular}{|c|c|c|c|c|c|c|}
\hline Adsorptive & $\begin{array}{c}\log \frac{H_{i}(\exp .)}{\mathrm{mol} / \mathbf{k g}} \\
{[-]}\end{array}$ & $\begin{array}{c}\log \frac{\mathrm{H}_{\mathbf{i}} \text { (th.) }}{\mathbf{m o l} / \mathbf{k g}} \\
{[-]}\end{array}$ & $\begin{array}{l}\mathbf{M}_{0}^{\mathbf{X}_{0}} \\
{\left[\AA^{2}\right]}\end{array}$ & $\begin{array}{c}\mathbf{M X}{ }_{2} \\
{\left[10^{-38} \mathrm{C}^{2} / \AA^{4}\right]}\end{array}$ & $\begin{array}{c}\mathbf{M}^{\mathbf{X}}{ }_{\text {acc }} \\
{\left[10^{-19} \mathrm{C}\right]}\end{array}$ & $\begin{array}{c}\mathbf{M}^{\mathbf{X}}{ }_{\text {don }} \\
{\left[10^{-19} \mathrm{C}\right]}\end{array}$ \\
\hline Acetic Acid & $-0,65$ & $-1,11$ & 92,26 & 184,80 & 3,16 & 6,14 \\
\hline Aniline & 0,87 & 0,69 & 134,93 & 164,13 & 1,91 & 1,79 \\
\hline Benzoic Acid & 0,96 & 1,56 & 152,86 & 195,94 & 2,18 & 6,42 \\
\hline 1-Butanol & 0,87 & 0,30 & 128,36 & 136,05 & 6,44 & 2,95 \\
\hline Butanone & 0,22 & 0,17 & 120,65 & 118,29 & 4,45 & 0,00 \\
\hline
\end{tabular}




\begin{tabular}{l|cccccc} 
Butyric Acid & 0,69 & 0,68 & 130,91 & 178,99 & 3,03 & 5,94 \\
o-Cresole & 2,17 & 2,12 & 148,66 & 159,87 & 0,88 & 5,56 \\
p-Cresole & 2,17 & 2,03 & 149,96 & 164,11 & 1,33 & 5,61 \\
Cis-1,2-Dichloroethene & 1,74 & 1,44 & 111,66 & 68,97 & 0,00 & 0,00 \\
Ethanol & $-1,82$ & $-1,49$ & 88,27 & 134,79 & 6,70 & 2,92 \\
Methanol & $-2,78$ & $-2,42$ & 67,56 & 136,54 & 6,83 & 3,11 \\
Nitrobenzene & 1,39 & 1,85 & 149,38 & 134,69 & 0,38 & 0,00 \\
1-Octanol & 3,91 & 3,80 & 208,71 & 141,85 & 5,99 & 3,03 \\
Phenol & 1,74 & 1,26 & 131,12 & 165,18 & 1,11 & 5,83 \\
1-Propanol & $-0,74$ & $-0,56$ & 108,34 & 134,43 & 6,46 & 2,95 \\
2-Propanol & $-0,74$ & $-0,75$ & 106,44 & 131,99 & 6,70 & 2,24 \\
Propanone & $-0,30$ & $-0,70$ & 102,68 & 122,98 & 4,47 & 0,00 \\
Propionic Acid & $-0,65$ & $-0,21$ & 110,82 & 179,07 & 3,11 & 5,96 \\
Propionitrile & $-0,39$ & $-0,27$ & 102,28 & 120,93 & 2,10 & 0,00 \\
Pyridine & $-0,10$ & $-0,19$ & 116,98 & 120,21 & 5,43 & 0,00 \\
Tetrachloroethene & 3,47 & 3,76 & 147,45 & 27,16 & 0,00 & 0,00 \\
Toluene & 2,61 & 2,65 & 140,57 & 71,77 & 0,00 & 0,00 \\
Trichloroethene & 2,61 & 2,66 & 130,91 & 47,75 & 0,00 & 0,00 \\
Trichloromethane & 1,82 & 1,70 & 117,53 & 68,77 & 0,00 & 0,00
\end{tabular}

Standard deviation: 0,347

As a first step we now calculated the 7 -moments for each adsorbate, corresponding to $\mathrm{i}=-2, \ldots, 4$ in eqs. 11 and 12 (see Tables 1 and 2). This was done using the program COSMOtherm_3.0, based on DFT-COSMO calculations from TURBOMOLE using a TZVP-basis set and the BP-functional (Schäfer (2000)). Typical calculation times for the small molecules considered in this study are $30 \mathrm{~min}$. on an INTEL-PII-600MHz computer.

In the case of the adsorption from gas-phase we first performed a direct $\sigma$-moment regression, yielding the equation:

$$
\log \left(H e_{\text {gas }}^{X}\right)=-8.663+0.0630 \cdot M_{0}^{X}+0.0225 \cdot M_{2}^{X}+0.597 \cdot M_{d o n}^{X}
$$

It turned out, that the $3^{\text {rd }}$ and higher moments as well as the acceptor moment were not significant. The results are shown in Figure 4. 


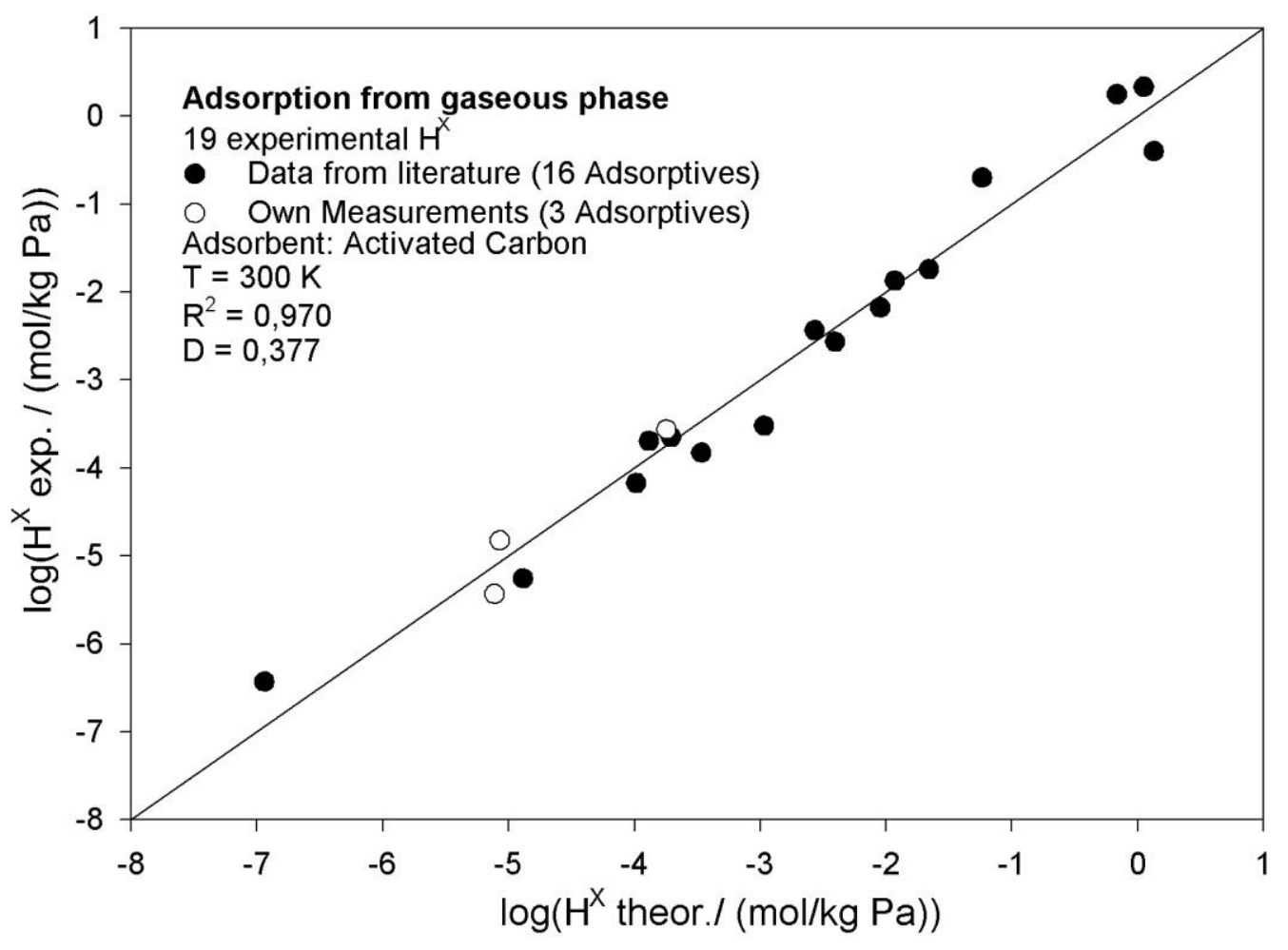

Figure 4: Correlation of adsorption data from gaseous phase with sigma moments

In the case of wet adsorption from water to $\mathrm{AC}$ we had experimental data for a broader range of compounds available. In a first $\sigma$-moment regression pyridine was clearly detected as an outlier. We therefore left it out in the final regression, yielding

$\ln \left(\mathrm{He}_{\text {water }}^{\mathrm{X}}\right)=-1.971+0.0430 \cdot \mathrm{M}_{0}^{\mathrm{X}}-0.0525 \cdot \mathrm{M}_{2}^{\mathrm{X}}-0.251 \cdot \mathrm{M}_{\mathrm{acc}}^{\mathrm{X}}+0.311 \cdot \mathrm{M}_{\mathrm{don}}^{\mathrm{X}}$

Again, addition of $3^{\text {rd }}$ and higher moments did not significantly improve the fit. The standard deviation of 0.35 log-units, which corresponds to an error of a factor 2.3 for the adsorption constants, still is safely within the experimental error, because the data were taken from literature and the experimental conditions (type of activated carbon, pretreatment of the activated carbon, temperature, equilibration time, etc.) of different data vary and the quality of the correlation can be increased by the use of a data basis that is taken with constant experimental conditions. For example, the deviation of pyridine was about 4.3 times the standard deviation, and thus an experimental error was highly likely, since pyridine usually is well described by COSMO-RS regarding other properties. Therefore we did our own measurement on 
pyridine, yielding to $\log \left(\mathrm{H}^{\text {Pyridine }}\right)=-0.096$, which corresponds to an error of a factor 0.28 times the standard deviation. The results are shown in Figure 5.

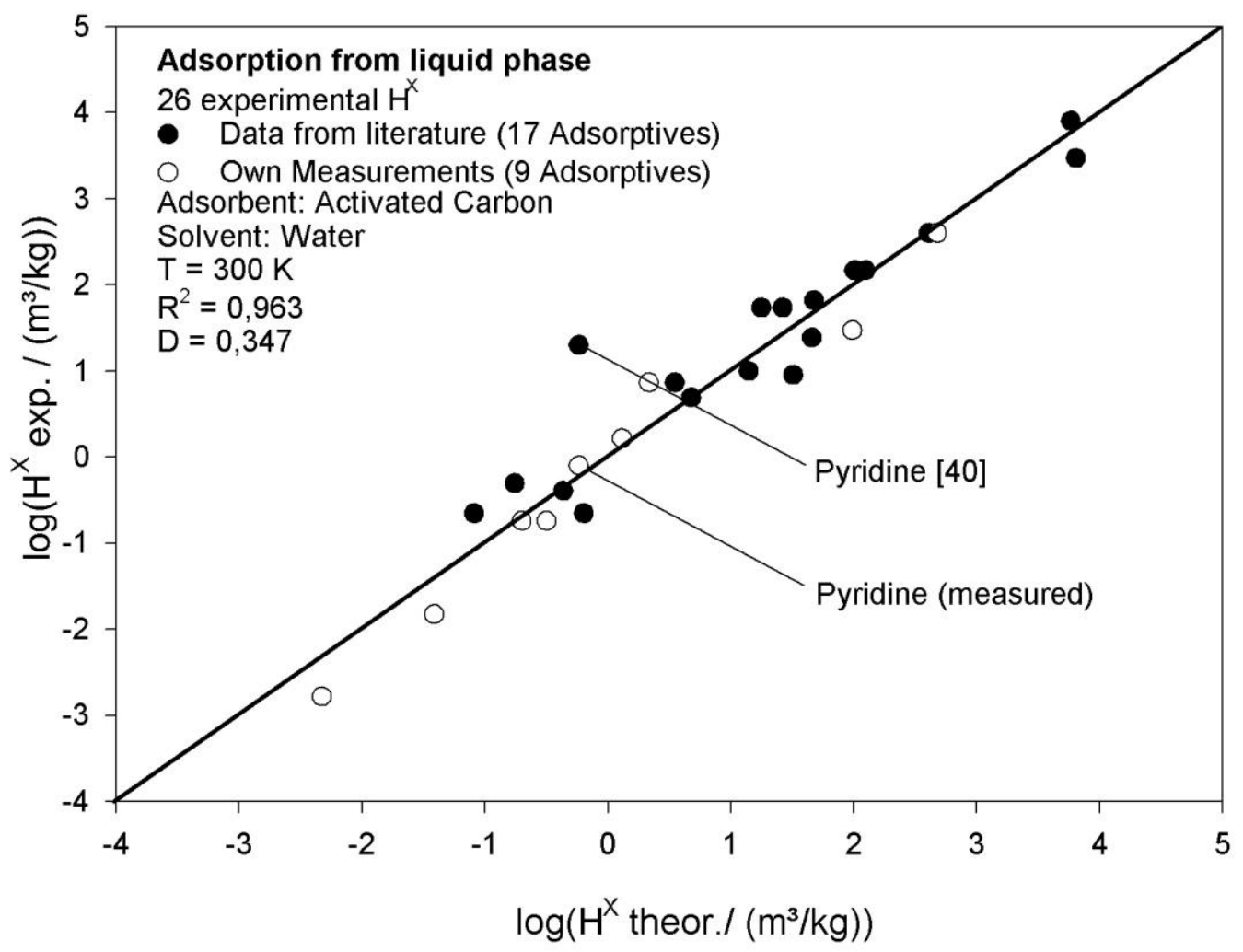

Figure 5: Correlation of adsorption data from aqueous phase with sigma moments

\section{Physical interpretation}

The regression parameters for the of the $\sigma$-moments include information about the difference of the interactions of the two involved phases, i.e. of gas phase and dry activated carbon in the first case, and about aqueous phase and wet activated carbon in the second case. [Note, that $\ln \left(\mathrm{H}^{\mathrm{X}}\right)$ is related to free energy by the factor $1 / \mathrm{RT}$, which is $1 / 2.5 \mathrm{~kJ} / \mathrm{mol}$ at room temperature.]

- Since $\mathrm{M}_{0} \mathrm{X}$ is the molecular area (in $\AA^{2}$ ), the coefficient of this moment takes into account all surface proportional interactions, which may be dispersion (or van der Waals) energies, cavitation energies, and various smaller contributions. The value of 0.0630 in the gas phase equation corresponds to $0.36 \mathrm{~kJ} / \mathrm{mol} / \AA^{2}$. The main contribution here is the dispersion energy, which is zero in the gas phase and 
about $-0.25 \mathrm{~kJ} / \mathrm{mol} / \AA^{2}$ in condensed phases. (Note that the adsorbate phase contributes with a negative sign. Hence we get a positive coefficient.) For the case of adsorption from water the dispersive energy contributions are almost identical in both phases, but the pure aqueous phase is known to have a high positive cavitation energy of about $0.2 \mathrm{~kJ} / \mathrm{mol} / \AA^{2}$. This matches quite well with the coefficient of $0.0430=0.24 \mathrm{~kJ} / \mathrm{mol} / \AA^{2}$.

- The second moment is a measure for electrostatic interactions. The positive coefficient in the first equation results from the fact, that the condensed phase, i.e. the adsorbate, is very well able to make favorable electrostatic interactions with the adsorbate, while there is no electrostatic interaction in the gas phase. On the other hand, the negative coefficient in the second case expresses the fact, that pure water makes even better electrostatic interactions than active carbon.

- The coefficient of the hydrogen-bond donor moment $\mathrm{Mdon}^{\mathrm{x}}$ expresses the ability of the phases to interact with donors, i.e. to act as acceptor. Hence the large positive coefficients in the both equations correspond to the fact, that activated carbon has a lot of acceptor capacity due to the oxygenated sites on the surface.

- Finally, the coefficient of the acceptor moment expresses donor capacity of the phases. In agreement with our expectation there is no significant donor capacity in activated carbon in the gas phase and hence the corresponding coefficient vanishes in the respective regression. In the liquid phase Franz (2000) detected the formation of water clusters around hydrophilic oxygen groups and ions on the surface of activated carbons. Thereby the equilibrium of donors and acceptors of the pure water is disturbed. As we expected wet activated carbon has a lower donor capacity than pure water, which leads to the negative contribution of the acceptor moment in eq. 18.

\section{Summary and Outlook}

It was shown, that the extension of COSMO-RS to the adsorption from gas phase and from aqueous solution is a promisingly way for the prediction of adsorption equilibria. The presented prediction method is based on $\sigma$-moments, which are directly derived from the polarization charge distributions from $a b$ initio calculations. Presently, this method is restricted to very dilute solutions. The characteristics of the 
solvent (or gas phase) and of the adsorbing solid are contained in the regression parameters. These parameters can be interpreted in a physically founded way.

As a next step we will try to make use of the information about the adsorbing solid, which is covered in the regression parameters, in order to derive an approximate $\sigma$ profile $p_{S 2}^{X}$ of the solid. With the knowledge of that $\sigma$-profile we should be able to extend the method to finite adsorbate concentrations using the standard COSMO-RS equations. 


\section{Nomenclature}

\begin{tabular}{|c|c|c|}
\hline aeff & {$\left[\AA^{2}\right]$} & Contact area \\
\hline c & {$\left[\mathrm{mol} \mathrm{l}^{-1}\right]$} & Equilibrium concentration \\
\hline$c_{S}^{i}$ & {$\left[\AA^{-2+2 i} C^{-1}\right]$} & Regression parameter of the i-th moment \\
\hline Chb & {$\left[\mathrm{kJ} \mathrm{mol}^{-1}\right]$} & Strength coefficient of the hydrogen bonding \\
\hline$E$ & {$[\mathrm{~kJ}]$} & Energy \\
\hline$f_{i}(\sigma)$ & {$\left[\mathrm{C} / \AA^{2 i}\right]$} & Profile-function of the i-th moment \\
\hline G & {$[\mathrm{kJ}]$} & Enthalpie \\
\hline $\mathrm{H}_{\text {gas }}$ & {$\left[\mathrm{mol} \mathrm{Pa}^{-1} \mathrm{~kg}^{-1}\right]$} & $\begin{array}{l}\text { Henry's Law coefficient for the adsorption from the gaseous } \\
\text { phase }\end{array}$ \\
\hline Hwater & {$\left[\mathrm{m}^{3} \mathrm{~kg}^{-1}\right]$} & $\begin{array}{l}\text { Henry's Law coefficient for the adsorption from the aqueous } \\
\text { phase }\end{array}$ \\
\hline $\mathrm{k}$ & {$\left[\mathrm{J} \mathrm{K}^{-1}\right]$} & Boltzmann constant \\
\hline K & {$[-]$} & Equilibrium constant \\
\hline$n$ & {$\left[\mathrm{~mol} \mathrm{~kg}^{-1}\right]$} & Mass-related amount of adsorbate \\
\hline$M_{i}$ & {$\left[C^{i} \AA^{2-2 i}\right]$} & i-th $\sigma$-Moment \\
\hline$\tilde{M}$ & {$\left[\mathrm{~kg} \mathrm{~mol}^{-1}\right]$} & Molecular mass \\
\hline $\mathrm{p}(\sigma)$ & {$\left[\AA^{4} \mathrm{C}^{-1}\right]$} & Frequency distribution of the charge (Sigma Profile) \\
\hline $\mathrm{R}$ & {$\left[\mathrm{kJ} \mathrm{mol}^{-1} \mathrm{~T}^{-1}\right]$} & General gas constant \\
\hline $\mathrm{T}$ & {$[\mathrm{K}]$} & Temperature \\
\hline$\alpha^{\prime}$ & {$\left[\mathrm{kJ} \mathrm{mol}{ }^{-1} \AA^{-2}\right]$} & Misfit energy coefficient \\
\hline$\mu \mathrm{s}(\sigma)$ & {$\left[\mathrm{kJ} \mathrm{mol}{ }^{-1} \AA^{2}\right]$} & Sigma Potential \\
\hline$\mu^{\mathrm{X}}$ & {$\left[\mathrm{kJ} \mathrm{mol}^{-1}\right]$} & Chemical potential \\
\hline$\rho$ & {$\left[\mathrm{kg} \mathrm{m}^{-3}\right]$} & Density \\
\hline$\sigma, \sigma^{\prime}$ & {$\left[C \AA^{-2}\right]$} & Charge \\
\hline
\end{tabular}

\section{Indices}
acc acceptor term
comb combinatorial term
don donor term 
hb Hydrogen bonding term

hydr hydration

$S \quad$ Solvent

\section{References}

Abraham, M.H., "ACD Approaches for phys. chem data prediction", Chem. Soc. Revs. 22,72 (1993)

Ahlrichs, R., Bär, M., Häser, M., Horn, H., and Kölmel, C., "Electronic Structure Calculations on Workstation Computers: the Program System TURBOMOLE ", Chem. Phys. Letters 162, 165 (1989)

Avdeef, A.; Berger, C. M.; Brownell, C., "pH-Metric Solubility. 2. Correlation Between the Acid-Base Titration and the Saturation ShakeFlask Solubility-pH Methods.", Pharmaceutical Research 17, 85 (2000)

Baldauf, G., Zimmer, G., "Adsorptive Entfernung leichtflüchtiger Halogenkohlenwasserstoffe bei der Wasseraufbereitung ", Vom Wasser 66, 21-31 (1986)

Busalla, T., "Berechnung von Membranverteilungskoeffizienten" Diploma Thesis, Univ. Cologne (1996)

Chatzopoulos, D., Varma, A., "Activated Carbon Adsorption and Desorption of Toluene in the Aqueous Phase ", AlChE Journal 39, 2027 (1993)

Clausen, I., Experimentelle und theoretische Analyse der Anwendbarkeit von COSMO-RS für Verteilungskoeffizienten, Ph-D thesis, TU Berlin (1998)

CLOGP program, BioByte Corporation, Claremont, CA

COSMOtherm program, COSMOlogic GmbH\&CoKG, Leverkusen, Germany, 1999

Cramer, C.J., Truhlar, D.G., In Lipkowitz, K.B., and Boyd, D.B., (Eds.), "Implicit Solvation Models: Equilibria, Structur, Spectra and Dynamics" Reviews in Computational Chemistry 6, VCH Publishers, New York, NY 
$\mathrm{DMol}^{3}$ program, Molecular Simulations, San Diego, CA

Fukuchi, K., Arai, Y., "Measurement and correlation of adsorption equilibria of single organic solute from dilute aqueous solution on activated carbon“, Kagaku Kogaku Ronbunshu 12, 603 (1986)

Fukuchi, K., Arai, Y., "Measurement and Prediction of Adsorption Equilibria of Organic Solutes from Dilute Aqueous Solutions on Activated Carbon ", Colloids and Surfaces 37, 387 (1989)

GAUSSIAN98 program, Gaussian Inc.

Gmehling, J., "Present status of group-contribution methods for the synthesis and design of chemical processes", Fluid Phase Equilibria 144, 37 (1998)

Gregg, S.J., Sing, K.S.W., Adsorption, Surface Area and Porosity, Academic Press, New York (1982)

Hansch,C., Leo,A.J., Substituent Parameters for Correlation Analysis in Chemistry and Biology, Wiley, New York, NY (1979)

Jorgensen , W. L. , "Computation of Free Energy Changes in Solution", In P. V.R. Schleyer, and Allinger, L., (Eds.) Encyclopedia of Computational Chemistry Vol. 2, Wiley, New York, NY 1061 (1998)

Juang, R.S., Wu, F.C., Tseng, R.L., "Adsorption Isotherms of Phenolic Compounds from Aqueous Solutions onto Activated Carbon Fibers", J. Chem. Eng. Data 41, 487 (1996)

Klamt , A. , "COSMO and COSMO-RS", In Schleyer, P. v.R. , and Allinger, L., (Eds.) Encyclopedia of Computational Chemistry Vol. 2, Wiley, New York, NY, 604 (1998)

Klamt, A., "Conductor-like Screening Model for Real Solvents: A New Approach to the Quantitative Calculation of Solvation Phenomena", J. Phys. Chem. 99, 2224 (1995)

Klamt, A., Eckert, F., " COSMO-RS: a novel and efficient method for the a priori prediction of thermophysical data of liquids" Fluid Phase Equilibria 172, 43 (2000) 
Klamt, A., Eckert, F., Fox, T., Hasselbach, K., (to be published)

Klamt, A., Eckert, F., Hornig, M., (to be published)

Klamt, A., Eckert, F., Hornig, M., Blake, J.F., Perspectives in Drug Design and Development (to be published)

Klamt, A., Jonas, V., Buerger, T., Lohrenz, J.C.W. , " Refinement and Parametrization of COSMO-RS " J. Phys. Chem. 102, 5074 (1998)

Klamt, A.; Schüürmann,G., "COSMO: A New Approach to Dielectric Screening in Solvents with Explict Expressions for the Screening Energy and its Gradient “, J. Chem. Soc. Perkin Trans.2 , 799 (1993)

M. Franz, H. Arafat, N. Pinto, "Effect of the chemical surface heterogenity on the adsorption mechanism of sissolved aromatics on activated carbon", Carbon 38, 1807 (2000)

Maurer, S., Mersmann, A., Peukert, W., The Second Pacific Basin Conference on Adsorption Science and Technology, 14. - 18. May 2000, Brisbane, Australien

Maurer, S., Prediction of Single-Component Adsorption Equilibria, $\mathrm{Ph}-\mathrm{D}$ thesis, TU München (2000)

McFarland, J.W., Personal Communication (2001)

Miyahara, M., Okazaki, M., "Concentration Dependence of Surface Diffusivity of Nitrobenzene and Benzonitrile in Liquid Phase Adsorption onto an Activated Carbon ", J. Chem. Eng. Japan 25, 408 (1992)

MOPAC2000 program, Fujitsu, Japan, 1999

Radke, C.J., Prausnitz, J.M., "Adsorption of Organic Solutes from Dilute Aqueous Solution on Activated Carbon ", Ind. Eng. Chem. Fundam. 11, 445 (1972)

Sakoda, A., Kawazoe, K., Suzuki, M., "Adsorption of Tri- and Tetrachlorethylene from Aqueous Solutions on Activated Carbon Fibers ", Water Res. 21, 717 (1986)

Schäfer, A., Klamt, A., Sattel, D., Lohrenz, J.C.W., and Eckert, F., "COSMO Implementation in TURBOMOLE: Extension of an efficient quantum chemical code towards liquid systems", Phys. Chem. Chem. 
Phys. 2, 2187 (2000)

Schäfer, H., van Gunsteren, W.F. , Mark, A.E, "Estimating relative free energies from a single ensemble: Hydration free energies" J. Comp.

Chem. 20, 1604 (1999)

Sontheimer, H., Frick, B., Fettig, J., Hörner, G., Hubele, C., Zimmer, G. , Adsorptionsverfahren zur Wasserreinigung, Engler-Bunte-Institut der Universität Karlsruhe (1985)

Tomasi, J., Persico, M., "Molecular Interactions in Solution: An Overview of Methods Based on Continuous Distributions of the Solvent", Chem. Rev. 94, 2027 (1994)

Urano, K., Koichi, Y., Yamamoto, E., "Equilibria for Adsorption of Organic Compounds on Activated Carbons in Aqueous Solutions ", J. of Coll. and Interface Sci. 86, 43 (1982)

Yenkie, M.K.N., Natarajan, G.S., "Adsorption Equilibrium Studies of Some Aqueous Aromatic Pollutants on Granular Activated Carbon Samples", Separation Sci. and Tech. 26, 661 (1991) 